\title{
Surgical repair of a giant congenital left atrial aneurysm presenting with respiratory distress in a neonate
}

\author{
Alwaleed Al-Dairy ${ }^{1}$, hazem aljasem ${ }^{1}$, and Samir Srour ${ }^{1}$ \\ ${ }^{1}$ Damascus University Faculty of Medicine
}

January 15, 2021

\begin{abstract}
Congenital left atrial aneurysm is a very rare congenital cardiac anomaly, and very few cases have been reported with neonatal presentation. Despite the congenital origin of this condition, it may remain asymptomatic and patients may not present until their third decade of life. Even in asymptomatic cases, prompt surgical intervention is indicated for the prevention of fatal thromboembolic events such as stroke. In neonates and infants presenting early in life, surgical intervention is urgent to relieve respiratory distress symptoms. Herein, we introduce the case of a 3-week-old neonate who presented with severe respiratory distress symptoms requiring prompt intubation and mechanical ventilation. Diagnosis of congenital left atrial aneurysm was established and confirmed by transthoracic echocardiography and computed tomography angiography. The patient underwent surgical resection of the aneurysm using cardiopulmonary bypass and cardiac arrest. The patient was discharged from the hospital after three weeks with normal echocardiography.
\end{abstract}

\section{Surgical repair of a giant congenital left atrial aneurysm presenting with respiratory distress in a neonate}

Alwaleed Al-Dairy ${ }^{1,[?]}$, MD, Hazem Aljasem², MRCS, Samir Srour ${ }^{3}$, MD.

${ }^{1}$ Assistant Professor in Cardiac Surgery at Faculty of Medicine, Damascus University, ${ }^{2}$ Cardiac surgeon at Damascus University cardiac surgery hospital, ${ }^{3}$ Professor in pediatric cardiology at Faculty of Medicine, Damascus University.

*Correspondence to : Alwaleed Al-Dairy: Assistant Professor in Cardiac Surgery at Faculty of Medicine, Damascus University, and Cardiac Surgeon at Damascus University Cardiac Surgery Hospital, Damascus, Syria.

Email: alwaleedaldairy.aa@gmail.com; Tel+963112133545, Fax +9632129437.

ORCID: Alwaleed Al-Dairy (0000-0002-2239-646X).

\section{Abstract}

Congenital left atrial aneurysm is a very rare congenital cardiac anomaly, and very few cases have been reported with neonatal presentation. Despite the congenital origin of this condition, it may remain asymptomatic and patients may not present until their third decade of life. Even in asymptomatic cases, prompt surgical intervention is indicated for the prevention of fatal thromboembolic events such as stroke. In neonates and infants presenting early in life, surgical intervention is urgent to relieve respiratory distress symptoms. Herein, we introduce the case of a 3 -week-old neonate who presented with severe respiratory distress symptoms requiring prompt intubation and mechanical ventilation. Diagnosis of congenital left atrial aneurysm was established and confirmed by transthoracic echocardiography and computed tomography angiography. 
The patient underwent surgical resection of the aneurysm using cardiopulmonary bypass and cardiac arrest. The patient was discharged from the hospital after three weeks with normal echocardiography.

\section{Introduction :}

Congenital left atrial aneurysm (CLAA) is an extremely rare congenital cardiac anomaly with potential serious complications on long-term basis such as arrhythmias, thromboembolic events and myocardial dysfunction (1,2). The first description of CLAA was introduced by Semans and Taussig in 1938 (3). Growth of CLAA may be attributed to dysplasia of the pectinate muscles which leads to poor myocardial contractility of the left atrium (LA). This results in progressive dilatation of LA according to laplace law $(2,4,5)$. It is usually an isolated lesion, and the most common associated lesion is secondary mitral valve regurgitation $(1,2)$. It has been reported that diagnosis of CLAA depends on its origin from a normal LA and its location within the pericardium with well-defined communication with LA (6). The diagnostic modalities of CLAA consist of non-invasive imaging such as transthoracic or transesophageal echocardiography (TTE, TEE), computed tomography angiography(CTA), and magnetic resonance imaging (MRI) (1,3). Despite the congenital origin of this condition, it may remain asymptomatic and patients may not present until their third decade of life, with very few reported cases of neonatal presentation $(2,4)$. Even in asymptomatic cases, prompt surgical intervention is indicated, for the prevention of fatal thromboembolic events such as stroke $(1,2,7)$. In neonates and infants presenting early in life, surgical intervention is urgent to relieve respiratory distress symptoms (8). Herein we present a case of a 3-week-old neonate who presented with respiratory distress, and was diagnosed to have a giant CLAA. Successful surgical resection of the CLAA was performed as soon as the diagnosis was established.

\section{Case presentation:}

A 3-week-old neonate weighing 3400 grams, was brought to the emergency department of a pediatric hospital. The neonate who was delivered with no relevant birth details, has recently suffered from severe respiratory distress symptoms, and was admitted and promptly intubated in that hospital. Chest-x ray (CXR) showed a well-delineated (mass) on the left border of the heart extending to the left hemithorax, and causing complete compression of the left inferior pulmonary lobe (Figure-1). TTE revealed massive dilatation of LA (LA aneurysm) about $8 \mathrm{~cm}$ with moderate to severe mitral regurgitation (MR). CTA was performed to precisely evaluate the LA aneurysm, its relation with the left ventricle (LV), and the condition of the pulmonary veins and other surrounding structures (Figure-2-A, Figure-2-B). Depending on the clinical state of the patient, surgical intervention was scheduled on urgent basis. Median sternotomy approach was used. Upon opening the pericardium, the heart protruded outside the chest, and the LA appendage was apparent and intact (it was not part of the aneurysm) (Figure-3). Total cardiopulmonary bypass (CPB) with bicaval cannulation was prepared, and the heart was arrested by antegrade cold blood cardioplegia. The heart was lifted outside the pericardium and rightward (as in the repair of total anomalous pulmonary venous connection), and the aneurysm was found posteriorly and on the left side with very thin wall. The aneurysm was opened, and its communication with LA was confirmed (Figure-4). The left atrial appendage was not the origin of the aneurysm; however, it was originating from the posterior wall of the LA near the posterior mitral annulus. The aneurysm was of sessile nature, and extended to the posterior wall of LV adjacent to marginal arteries. It was completely resected, with special attention to avoid injury to the mitral annulus or any of the marginal arteries. The resulted defect was closed by two layers of running $7 / 0$ prolene suturing (Figure-5). The LA was opened as in mitral surgery, and mitral valve was inspected. The leaflets and subvalvar apparatus were normal. The valve was tested by saline test and was completely competent. Aortic cross clamp was released, and weaning off $\mathrm{CPB}$ was uneventful. The patient was discharged from the hospital after 21 days with significant clinical improvement, and normal echocardiography.

\section{Discussion:}

CLAA is a very rare entity in neonates with $70 \%$ of cases being left atrial appendage aneurysms, and the remainder are left atrial aneurysms (1). In neonates and infants, the early presentations of the lesion may be attributed to the secondary MR, and airway obstruction which may result in heart failure and respira- 
tory distress syndrome $(2,8)$. Even in asymptomatic patients, surgery is mandatory due to the potential life threatening complications, or the compression of the adjacent cardiac and respiratory structures $(2,5)$. According to previous reports, the risk of complications increases as the size of CLAA increases (9). Surgery is the treatment of choice and there are multiple surgical approaches including median sternotomy with or without $\mathrm{CPB}$, endoscopic resection, left thoracotomy, and mini thoracotomy $(4,6)$ The surgical intervention through median sternotomy and with CPB is the preferred one. This is because it enables the surgeon to perform the aneurysmectomy and mitral valve repair if there is any distortion of the valve (2).

To the best of our knowledge there are only four reported cases of neonatal surgical management of congenital left atrial aneurysms (one was LA aneurysm, and three were LA appendage aneurysms). Our case is very unique for two reasons: the first, it represents the smallest age at which CLAA was surgically managed, the second it is the largest LA aneurysm ever presenting in a neonate. Moreover, it had a sessile nature, and extended to the posterior wall of LV adjacent to marginal arteries.

\section{Conclusion:}

Giant CLAA is an extremely rare defect with potential lethal complications and significant morbidity. Early surgical management is mandatory to prevent devastating complications, and it is lifesaving in neonates presenting early in life with severe respiratory distress symptoms. Surgery for CLAA is safe, uncomplicated with complete regression of the symptoms even in neonates.

Consent: Written informed consent was obtained from the patient's parents for publication of this report and the images.

Conflict of interest statement: The authors have no conflict of interest.

Data availability statement: The data is available in the archive office at Damascus University cardiac surgery hospital.

Funding: None

Declaration : we declare that none of the authors listed on the manuscript are employed by a government agency that has a primary function other than research and/or education. Moreover, none of the authors is submitting this manuscript as an official representative or on behalf of the government.

\section{Figure legends:}

Figure-1. Preoperative CXR

Figure-2. Preoperative CT scan

Figure-3. Intraoperative image showing the protruded heart outside the chest and the (intact) LA appendage.

Figure-4. Intraoperative image showing the opened aneurysm

Figure-5. Intraoperative image showing the suture line of closing the defect after resection of the aneurysm

\section{References}

1- Kunishima T, Musha H, Yamamoto T, Aoyagi H, Kongoji K, Imai M, Ozawa A, So T, Nagashima J, Murayama M. Congenital giant aneurysm of the left atrial appendage mimicking pericardial absence case report. Jpn Circ J. 2001;65(1):56-59.

2- Chen Y, Mou Y, Jiang LJ, Hu SJ. Congenital giant left atrial appendage aneurysm: a case report. J Cardiothorac Surg. 2017;12(1):15.

3- Khaled A Shams. When the left atrium becomes a monster: a case report. European Heart Journal Case Reports . 2020; 4 (4): 1-4. 
4- Low ZK, Yap KH, Fortier MV, Nakao M. Congenital left atrial appendage aneurysm with unexpected course of left anterior descending coronary artery. Interact Cardiovasc Thorac Surg. 2020 Nov 22:ivaa267. doi: 10.1093/icvts/ivaa267. Epub ahead of print. PMID: 33221886.

5- Vagefi PA, Choudhry M, Hilgenberg AD. Excision of an aneurysm of the left atrial appendage. J Thorac Cardiovasc Surg. 2007;133(3):822-823.

6- Valentino MA, Al Danaf J, Morris R, Tecce MA. Giant left atrial appendage aneurysm: A case of mistaken identity. J Cardiol Cases. 2017;15(4):129-131.

7- Thuu LQ, Thuc N, Vinh BDA, Hoai DTP. Surgical Treatment of Left Atrial Appendage Aneurysm: A Case Report. May 2019. doi:10.25373/ctsnet.8064095

8- Clarke JR, Zvaigzne CG, Disler D, Giuffre RM, Rebeyka IM, Patton DJ. Giant left atrial appendage aneurysm in a neonate. World J Pediatr Congenit Heart Surg. 2012; 3(3):392-395.

9- Yakut K, Varan B, Erdoğan İ. Asymptomatic giant congenital left atrial aneurysm. Turk J Pediatr. 2019;61(1):117-119.

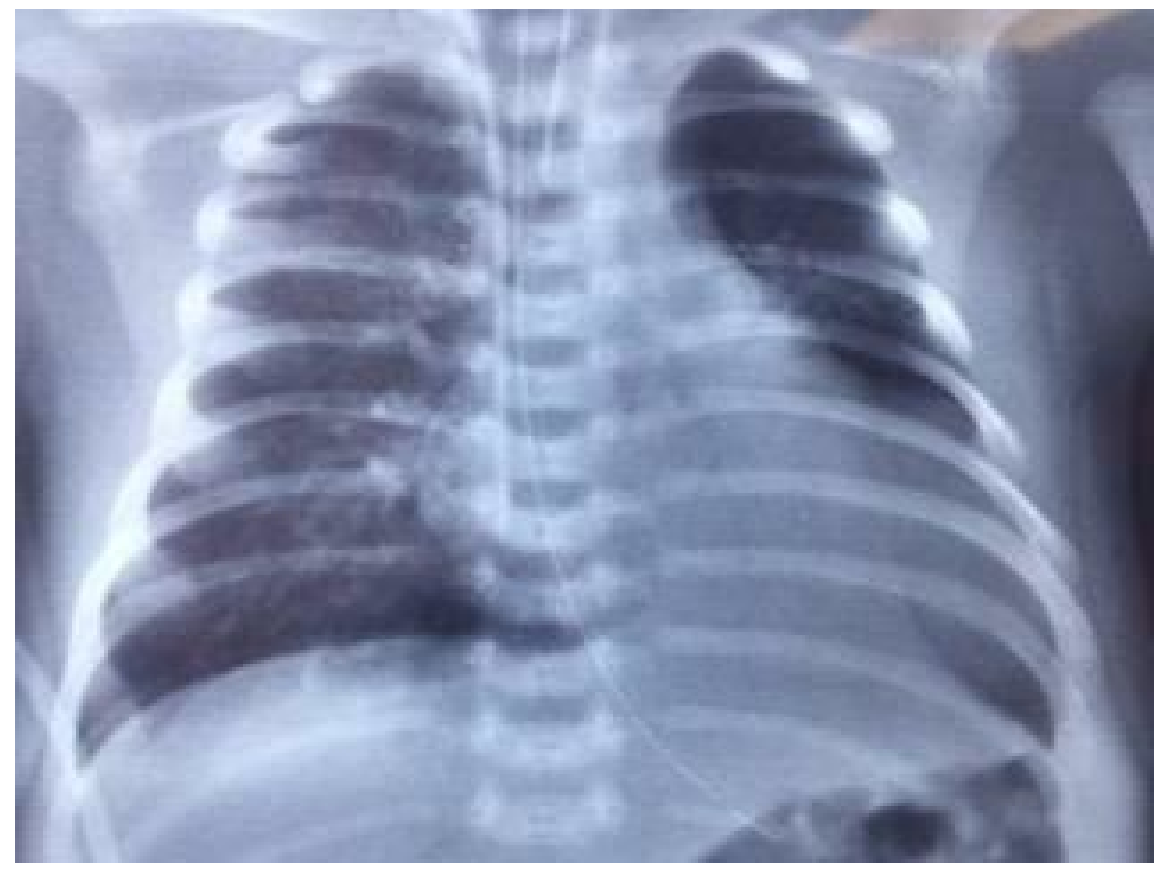



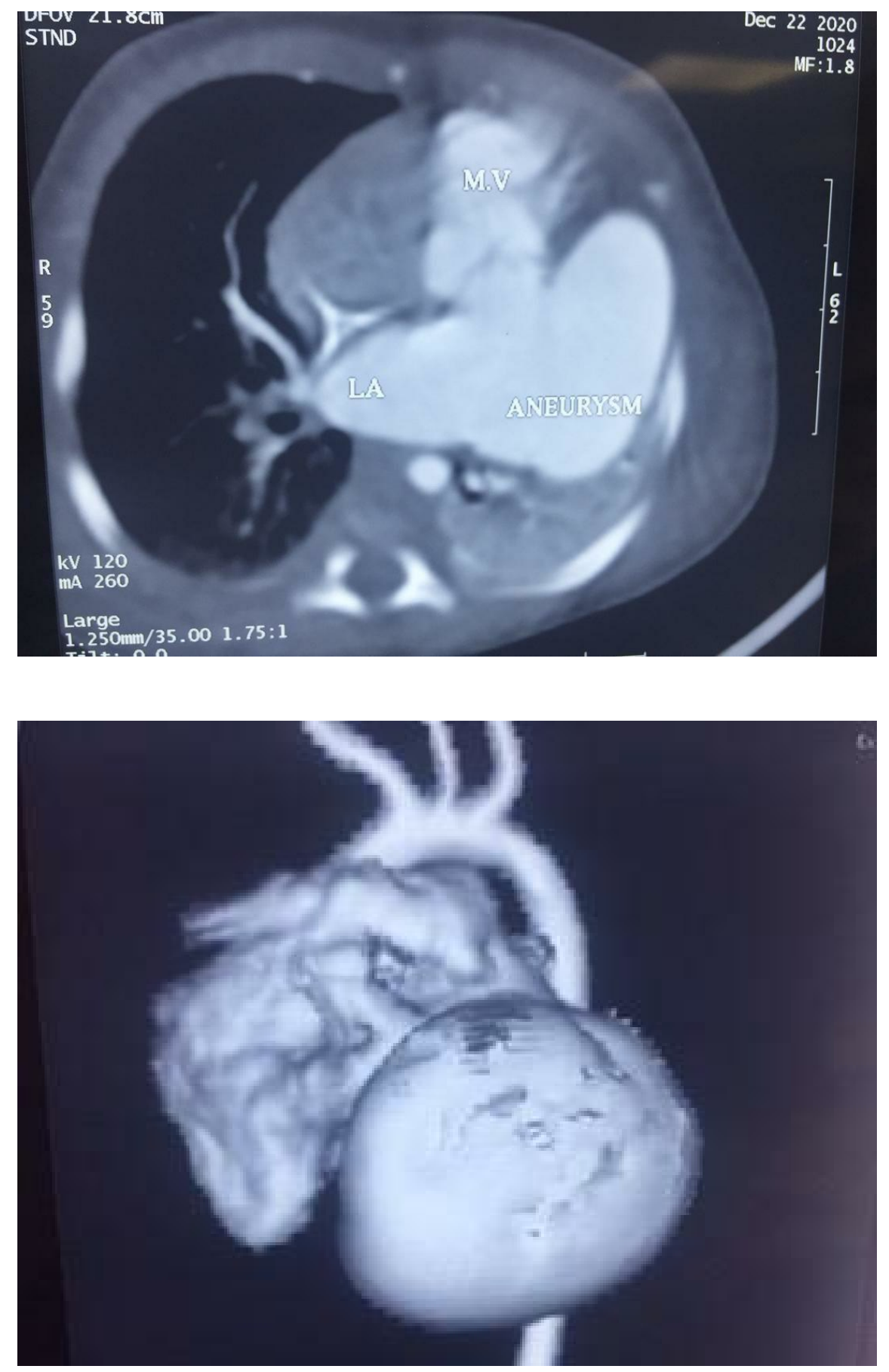


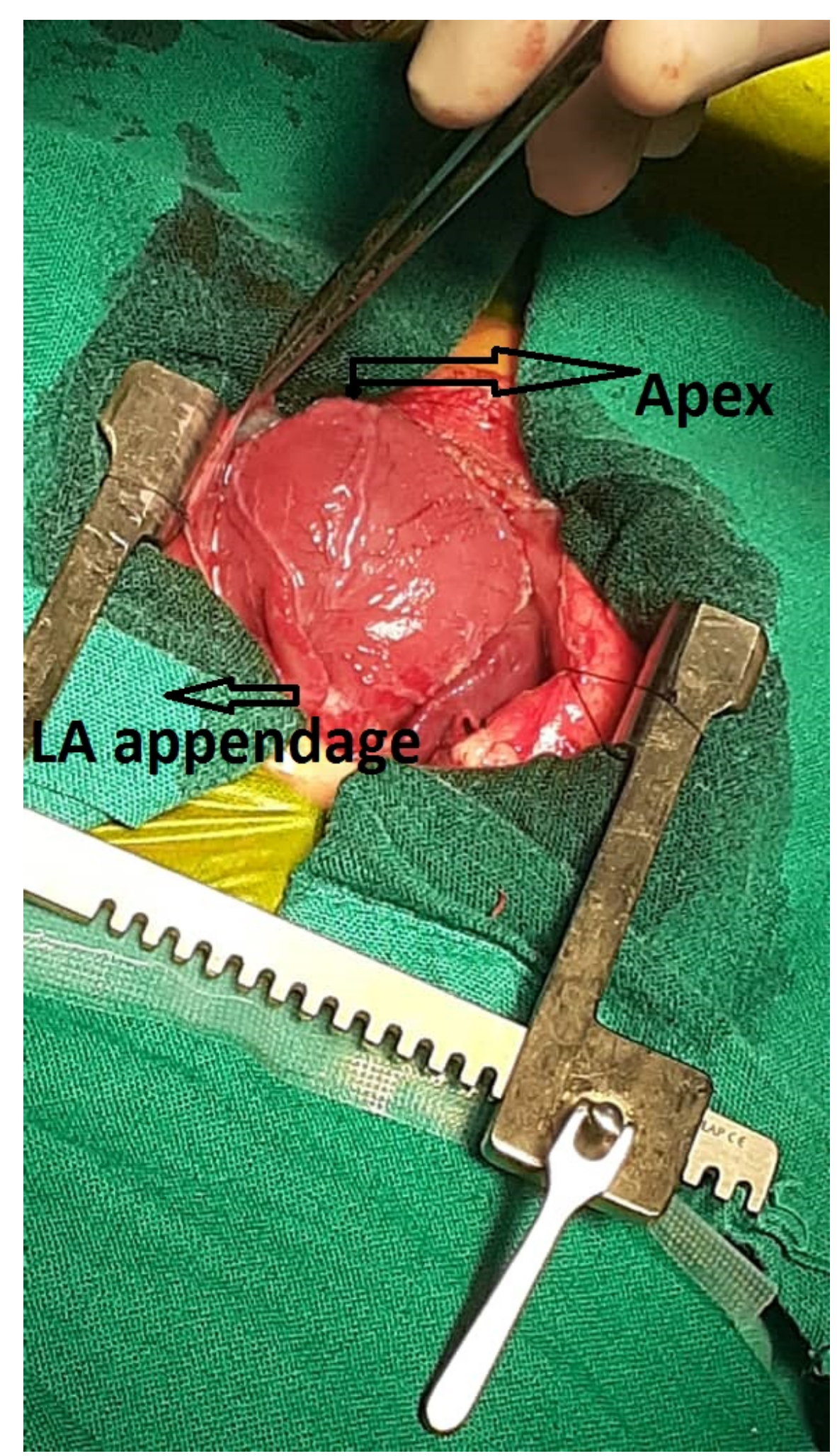




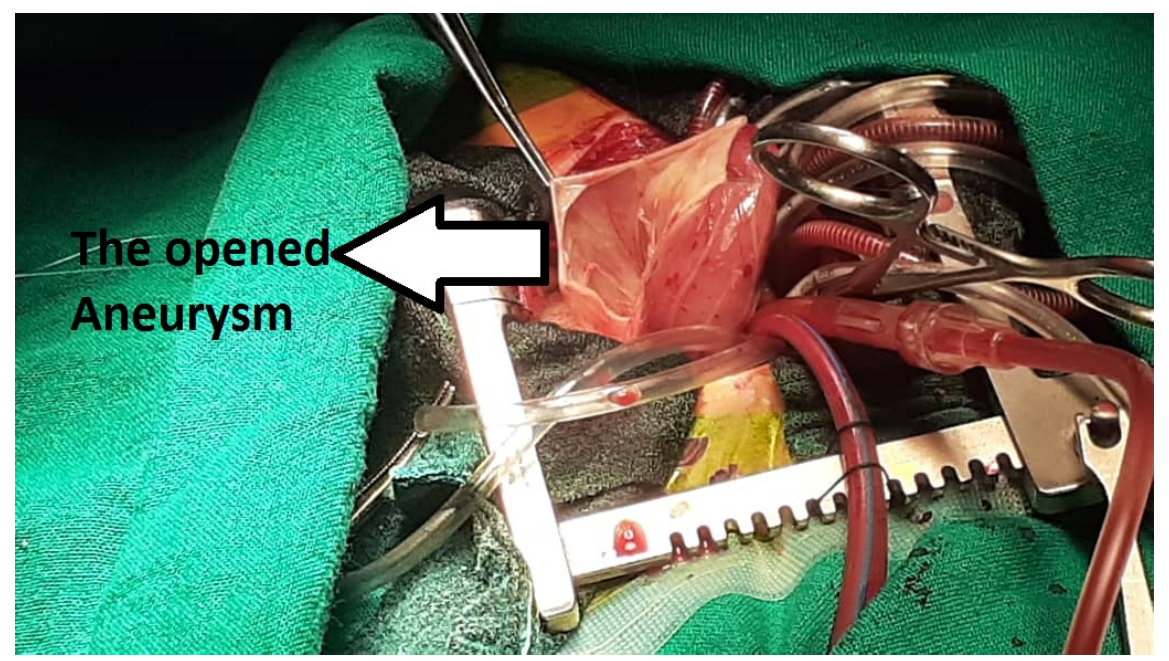




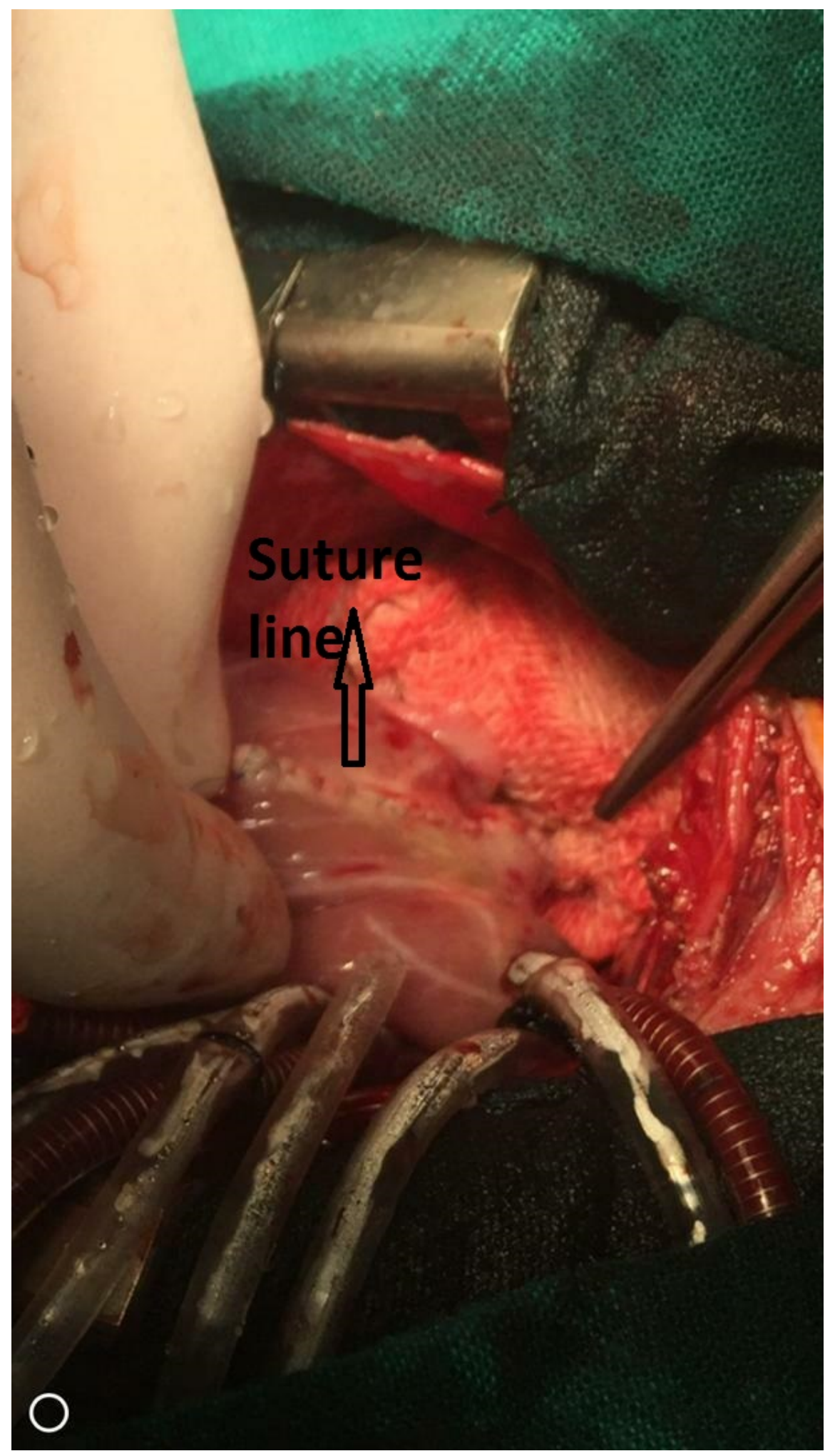

